\title{
Status of SuperNEMO experiment and last results of NEMO-3
}

\author{
Alberto Remoto* \\ on behalf of NEMO-3 and SuperNEMO collaborations \\ Laboratoire d'Annecy le Vieux de physique des particules, 9 Chemin de Bellevue 74941 \\ Annecy-le-Vieux CEDEX FRANCE \\ E-mail: remoto@in2p3.fr
}

\begin{abstract}
SuperNEMO is a next generation neutrino-less double beta decay experiment with a design capability to reach an half-life sensitivity of $10^{26} \mathrm{y}$ with $100 \mathrm{~kg}$ of ${ }^{82} \mathrm{Se}$, corresponding to an effective Majorana neutrino mass of $\left|m_{\beta \beta}\right|<0.04-0.10 \mathrm{eV}$. This detector has the unique capability to identify the two electrons from the double beta decay allowing to drastically reduce the background. Furthermore, it is able to measure all kinematical parameters of the event which could allow to determine the process leading to neutrino-less double beta decay. The status of SuperNEMO construction and the improvements expected with respect to the previous NEMO-3 experiment are presented. The R\&D started in 2007 and today all the requirements are achievable. The commissioning of one quarter of the tracker and one of the two calorimeters is expected by the end of this year in the Laboratoire Souterrain de Modane. The data taking is expected by the second half of 2016. The latest results from NEMO-3 are also presented. The experiment searched neutrino-less double beta decay from 2003 to 2011. Seven isotopes were studied with the unique combination of tracking and calorimetric measurement including $7 \mathrm{~kg}$ of ${ }^{100} \mathrm{Mo}$ and $1 \mathrm{~kg}$ of ${ }^{82} \mathrm{Se}$.
\end{abstract}

The European Physical Society Conference on High Energy Physics

22-29 July 2015

Vienna, Austria

\footnotetext{
*Speaker.

${ }^{\dagger}$ Supported by the Labex ENIGMASS, Investissements d’Avenir.
} 


\section{Introduction}

Experimental searches for neutrino-less double beta decay $(0 v \beta \beta)$ are one of the most active research topics in neutrino physics. The observation of such process is in fact of major importance since it will prove the Majorana nature of neutrinos and may give access to their absolute mass scale. The Majorana nature of the neutrino would have interesting implication in many extensions of the Standard Model of particle physics. For instance the see-saw mechanism requires the existence of a Majorana neutrino to explain naturally the smallness of neutrino masses. A Majorana neutrino would also provide a natural framework for the lepton number violation, and particularly for the leptogenesis process which may explain the observed matter-antimatter asymmetry of the Universe. The standard underlying mechanism providing a neutrino-less double beta decay is the exchange of a Majorana neutrino. In this case, the decay half-life can be written as:

$$
\left[T_{0 v}(A, Z)\right]^{-1}=G_{0 v}\left(Q_{\beta \beta}, Z\right)\left|M_{0 v}(A, Z)\right|^{2}\left|m_{\beta \beta}\right|^{2}
$$

where $\left|m_{\beta \beta}\right|$ is the effective neutrino mass, $M_{0 v}$ is the Nuclear Matrix Element (NME) and $G_{0 v}$ is the kinematical phase space factor. Other mechanisms could be involved in this process as the existence of right handed currents in the electroweak interaction, the exchange of super symmetric particles via the R-parity violating coupling, or extra dimensions and would results in a different topology of the final state. For a given mechanism and isotope, the $0 v \beta \beta$ decay half-life depends on the phase space factors and on the NME. While the former can be precisely calculated [1], the latter depends on the nuclear model adopted [2]. The decay half-lives of different isotopes can then differ by a few orders of magnitude with large theoretical uncertainties from NME calculations. Furthermore, the quenching of the axial coupling constant $g_{A}$ observed in single $-\beta$ and $2 v \beta \beta$ decay could increase half-lives $0 v \beta \beta$ as discussed in [3]. It is therefore essential to search for $0 v \beta \beta$ decays in several isotopes.

\section{The NEMO-3 experiment}

The NEMO-3 detector, shown in Fig. 1, performed precise measurement of double beta decay and searched neutrino-less double beta decay on seven isotopes, among which ${ }^{100} \mathrm{Mo}(\sim 7 \mathrm{~kg})$ and ${ }^{82} \mathrm{Se}(\sim 1 \mathrm{~kg})$. Smaller amount of other isotopes as ${ }^{130} \mathrm{Tl},{ }^{116} \mathrm{Cd},{ }^{150} \mathrm{Nd},{ }^{96} \mathrm{Zr}$ and ${ }^{48} \mathrm{Ca}$ were also present. The detector [4] had a cylindrical symmetry and was divided in 20 sectors. Each sector consisted in source planes containing the $\beta \beta$ emitting isotopes surrounded by a tracker and a calorimeter. The detector was installed in the Laboratoire Souterrain de Modane (LSM), under a rock overburden of 4800 m.w.e and took data between 2003 and 2011. The $\beta \beta$ events were emitted from thin source foils $\left(40-60 \mathrm{mg} / \mathrm{cm}^{2}\right)$ suspended between two concentric cylindrical tracking volumes. The tracker was composed by 6180 Geiger cells allowing a tracking precision of $\sigma_{t}=2-3 \mathrm{~mm}$ transversally to the cells and $\sigma_{z}=7-13 \mathrm{~mm}$ longitudinally. The tracking volume was surrounded by a segmented calorimeter made of 1940 large blocks of plastic scintillator coupled to low radioactivity 3" and 5" PMTs. The calorimeter provided a timing resolution of $\sigma=250 \mathrm{ps}$ while the energy resolution was $14-17 \%$ FWHM for $1 \mathrm{MeV}$ electrons. The detector was immersed in a $25 \mathrm{G}$ magnetic field to enhance charged particle identification, shielded from external gamma rays by $19 \mathrm{~cm}$ of low activity iron and from neutrons by $30 \mathrm{~cm}$ of water with boric 


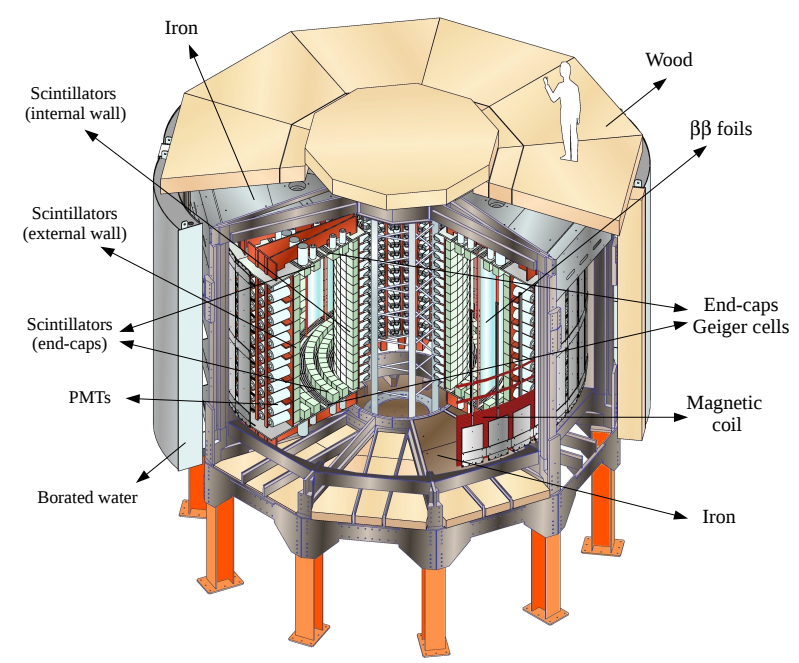

Figure 1: Schematic view of the NEMO-3 detector.

acid. After one year of data taking, a radon-free air facility flushing a tight tent surrounding the detector was installed to reduce the radon contamination in the tracking chamber by a factor 6 . The data are then divided in two data taking period, namely Phase I (before the anti-radon facility installation) with a live time of $1.02 \mathrm{y}$ and Phase II (after the anti-radon facility installation) with a live time of $3.94 \mathrm{y}$.

The absolute energy scale calibration was performed every 3 weeks with ${ }^{207} \mathrm{Bi}$ sources which provide internal conversion electrons of $482 \mathrm{keV}$ and $976 \mathrm{keV}$. For $99 \%$ of the PMTs the energy scale is known better than $2 \%$. The rare $1682 \mathrm{keV}$ internal conversion electron peak of ${ }^{207} \mathrm{Bi}$ was used to determine the systematic uncertainty on the energy scale which was found to be within $0.2 \%$. The main feature of NEMO-3 was its unique capability to fully reconstruct the kinematics of the events. This allows to perform topological selection of the events depending from the final states of interest, to reduce backgrounds and to discriminate among different mechanisms beyond the neutrino-less double beta decay [5].

\subsection{Background measurements}

The most important backgrounds for a $\beta \beta$ decay experiment come from the natural radioactivity of the detector materials due to the presence of long half-life isotopes, mainly ${ }^{238} \mathrm{U},{ }^{235} \mathrm{U},{ }^{232} \mathrm{Th}$ and ${ }^{40} \mathrm{~K}$ and their decay products. Since NEMO-3 detector was capable to identify different type of particles and event topologies, it has been possible to study the backgrounds combining tracking, calorimetric and timing information in different channels [6]. Activities of different background components are then obtained by adjusting Monte Carlo (MC) distributions to the data in different channels. The external backgrounds produced by the $\gamma$-ray flux entering the detector is determined by the detection of the electron produced by Compton effect in one of the scintillators, which cross the entire detector. The internal backgrounds due to contamination in the source foil is quantified looking for single $\beta$ emission or for $\beta-n \gamma$, if one or several $\gamma$ are emitted together with the electron. The radon contribution is determined studying the delayed $\beta-\alpha$ events produced by the ${ }^{214} \mathrm{Bi} \rightarrow{ }^{214} \mathrm{Po}$ cascade of ${ }^{222} \mathrm{Rn}$ chain. The background model developed for the $0 v \beta \beta$ search in 
the ${ }^{100} \mathrm{Mo}$ has been validated performing several tests. The ${ }^{208} \mathrm{Tl}$ activity was validated with two calibrated ${ }^{232} \mathrm{U}$ sources (decaying to ${ }^{208} \mathrm{Tl}$ ) which provide a $10 \%$ systematics uncertainty compared to HPGe activity measurements of the radioactive source. The ${ }^{214} \mathrm{Bi}$ activity was validated comparing the measurement obtained in the $1 e^{-} 1 \alpha$ and the $1 e^{-} N \gamma$ channels and have been found in agreement within $10 \%$ systematics. The background model was finally tested selecting $2 e^{-}$ events from the copper and tellurium foils where no double beta decay events are expected at high energy $\left(Q_{\beta \beta}\left({ }^{130} \mathrm{Te}\right)=2.53 \mathrm{MeV}\right)$. A very good agreement has been obtained with an exposure of $13.5 \mathrm{~kg} \times \mathrm{y}$, as shown in Fig. 2 (a).

\subsection{Measurements of $2 v \beta \beta$ in ${ }^{100} \mathrm{Mo}$}

The $2 v \beta \beta$ decay represents the irreducible background for the $0 v \beta \beta$ searches. The measurement of the $2 v \beta \beta$ is however interesting since it may provide information for the theoretical calculation of the NME and tests different nuclear models [7]. The analysis of the Phase I data provide the most precise direct measurement of the ${ }^{100} \mathrm{Mo} 2 v \beta \beta$ decay half-life corresponding to $T_{1 / 2}^{2 v}=7.16 \pm 0.01($ stat $) \pm 0.54($ syst $) \times 10^{18} \mathrm{y}$ [8]. More recently the analysis of Phase I and Phase II results in $T_{1 / 2}^{2 v}=6.93 \pm 0.04($ stat $) \times 10^{18} \mathrm{y}$ for $E_{2 e^{-}}>2 \mathrm{MeV}$ [9] . This value is in agreement with the previously published result and with the word average [10].

\subsection{Search for $0 v \beta \beta$ in ${ }^{100} \mathrm{Mo}$}

The search for $0 v \beta \beta$ decay for ${ }^{100}$ Mo has been performed on the total accumulated exposure of $34.3 \mathrm{~kg} \times \mathrm{y}$ [9]. The search has been focused in the $2.8-3.2 \mathrm{MeV}$ energy window, around the $Q_{\beta \beta}$ value of ${ }^{100} \mathrm{Mo}$. In this energy window the signal detection efficiency is $4.7 \%$.

No event excess has been observed above the background expectation and a limit for the halflife of $0 v \beta \beta$ decay is obtained using a modified frequentist analysis based on log-likelihood ratio test statistics [11]. Dedicated studies estimate the main systematic uncertainties to be $7 \%$ for the detection efficiency determined using dedicated runs with activity-calibrated ${ }^{207} \mathrm{Bi}$ sources. The uncertainty on $2 v \beta \beta$ is obtained from the fit to $2 e^{-}$events above $2 \mathrm{MeV}$ and is $0.7 \%$. The uncertainty on the ${ }^{214} \mathrm{Bi}$ and ${ }^{208} \mathrm{Tl}$ are estimated as described in the previous section and are found to be $10 \%$ respectively. The information of the binned energy sum distribution in the $2.0-3.2 \mathrm{MeV}$ energy window is used for signal and background (Fig. 2 (b)) together with the statistical and systematic uncertainties and their correlations as described in [11]. The background-only hypothesis is accepted with a p-value of $64.7 \%$. The half-life limit for the neutrino mass mechanism is $T_{1 / 2}^{0 v}>1.1 \times 10^{24}$ y $\left(90 \%\right.$ C.L.), which corresponds to $\left|m_{\beta \beta}\right|<0.33-0.62 \mathrm{eV}$, depending on the NME [2] and the phase space calculations [1]. Limits on other lepton number violating mechanisms have been also obtained in [9]. All results obtained for the different mechanisms by NEMO-3 are comparable with the best current results obtained with other isotopes.

It is worth to notice that no events have been observed in the $3.2-10 \mathrm{MeV}$ energy window for NEMO-3 sources containing isotopes with $Q_{\beta \beta}$ lower than $3.2 \mathrm{MeV}$ (i.e. ${ }^{100} \mathrm{Mo},{ }^{82} \mathrm{Se},{ }^{130} \mathrm{Te}$ and ${ }^{116} \mathrm{Cd}$ ) or without $\beta \beta$ emitters $(\mathrm{Cu})$ during the entire running period, which corresponds to an exposure of $47 \mathrm{~kg} \times \mathrm{y}$. The $\beta \beta$ decay has also been studied for the other isotopes in the detector but only partial exposures have been published. The NEMO-3 collaboration is currently finalising the analysis of the full exposure for all the isotopes. 


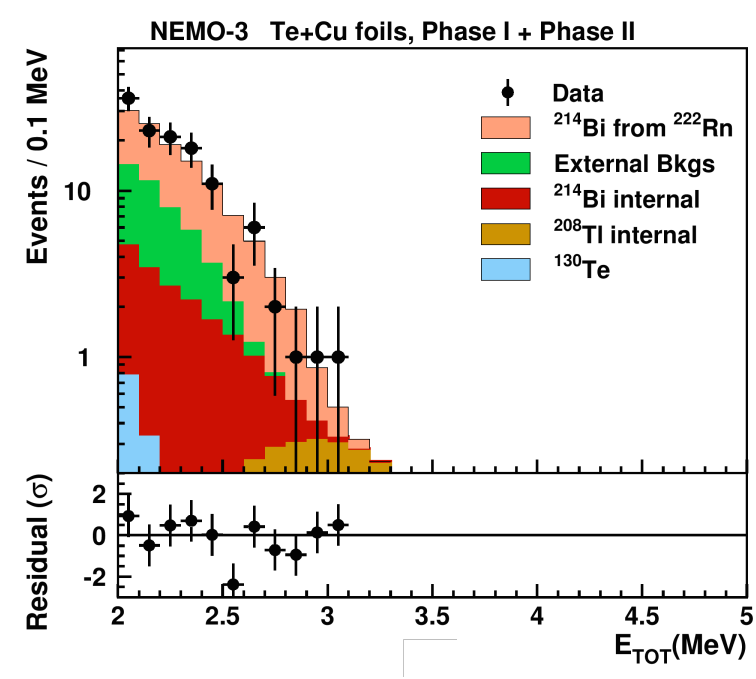

(a)

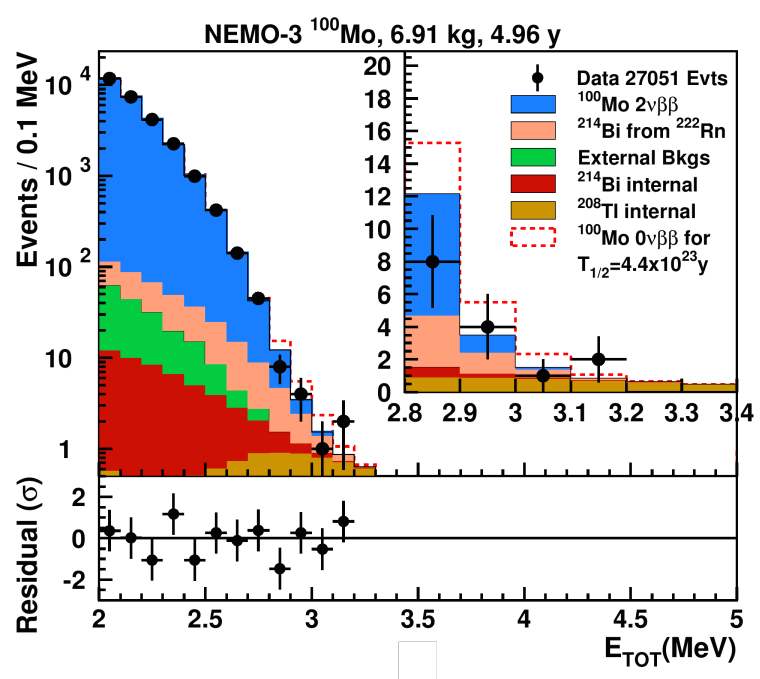

(b)

Figure 2: (a) Validation of the background model in the $2 e^{-}$channel from the copper and tellurium foils. (b) Distribution of the $2 e^{-}$energy sum of ${ }^{100} \mathrm{Mo}$ above $2 \mathrm{MeV}$. The ratio between the observed and the expected distributions from MC simulations are also shown [9].

\section{The SuperNEMO experiment}

SuperNEMO is the next generation $0 v \beta \beta$ experiment based on the NEMO-3 technique combining tracking and calorimetry. The goal is to gain 2 orders of magnitude in the current half-life sensitivity $T_{1 / 2}^{0 v}>1 \times 10^{26}$ y corresponding to effective neutrino mass of $\left|m_{\beta \beta}\right|<0.04-0.10 \mathrm{eV}$. SuperNEMO will consist of 20 identical planar modules housing each $5 \mathrm{~kg}$ of double beta decay isotope. The ${ }^{82} \mathrm{Se}$ is the baseline isotope given the ease to enrich and purify high masses by actual means in a reasonable time. The double beta decay sources will be shaped in thin foil made of selenium powder, polyvinyl-alcohol glue and thin mylar film.

A dedicated R\&D allowed to reduce the calorimeter energy resolution down to $4 \%$ FWHM at 3 $\mathrm{MeV}$. A particular effort has been made to reduce the radon emanation of the material and to prevent external radon penetration in the detector volume. The collaboration is currently building the first module called demonstrator which will observe $7 \mathrm{~kg}$ of ${ }^{82} \mathrm{Se}$. The demonstrator aims to register no background events in the $0 v \beta \beta$ energy region after $2.5 \mathrm{y}$. This will validate the choice of the materials adopted to reduce the background contamination and will validate the physics potential of the full SuperNEMO detector. The half-life sensitivity of the demonstrator module is expected to be $T_{1 / 2}^{0 v}>6.5 \times 10^{24}$ y leading to a mass limit $\left|m_{\beta \beta}\right|<0.20-0.40 \mathrm{eV}$ (90\% C.L.) which improves by factor 6 the current limit obtained by NEMO-3 with ${ }^{100}$ Mo.

The pure iron mechanical structure and the clean tend for detector assembling have been installed in the LSM. The first demonstrator calorimeter is under construction with the assembly of the polystyrene scintillators with the 8" Hamamatsu PMTs. The first quarter of the tracker has been assembled, commissioned at sea level and it is currently being transported to the LSM. The second quarter of the tracker is currently under assembly. A total of $5.5 \mathrm{~kg}$ of ${ }^{82} \mathrm{Se}$ have been purchased 
and purified. The source fabrication materials have been selected and the two first strips of the source foils are under measurement in the BiPo detector. The commissioning of the first quarter of the tracker and one of the two calorimeters is expected by the end of this year. The data taking is expected by the second half of 2016 .

\section{Summary}

The NEMO-3 experiment was unique in the direct reconstruction of the full signature of $0 v \beta \beta$ decays with powerful background rejection capabilities. With an exposure of $34.3 \mathrm{~kg} \times \mathrm{y}$, no evidence for the $0 v \beta \beta$ decay of ${ }^{100}$ Mo has been found. Taking into account statistical and systematic uncertainties, the half-life limit for the light Majorana neutrino mass mechanism is $T_{1 / 2}^{0 v}>1.1 \times 10^{24}$ y $(90 \%$ C.L.) which corresponds to a limit on the effective Majorana neutrino mass of $\left|m_{\beta \beta}\right|<0.33-0.62 \mathrm{eV}$. The absence of events in the high energy part of the spectrum is an encouraging result for future experiments based on the tracking+calorimetry technique planning to use high $\mathrm{Q}_{\beta \beta}$ isotopes such as ${ }^{48} \mathrm{Ca},{ }^{96} \mathrm{Zr}$ and ${ }^{150} \mathrm{Nd}$.

SuperNEMO is the next generation experiment and the first demonstrator module is currently under construction in the LSM. No background event is expected in the $0 v \beta \beta$ region in 2.5 years for $7 \mathrm{~kg}$ of ${ }^{82} \mathrm{Se}$. The half-life sensitivity is expected to be $T_{1 / 2}^{0 v}>6.5 \times 10^{24}$ y corresponding to an effective mass sensitivity $\left|m_{\beta \beta}\right|<0.2-0.4 \mathrm{eV}$ (90\% C.L.). Physics data taking of the demonstrator is expected to start in the second half of 2016.

\section{References}

[1] J. Kotila and F. Iachello, Phys. Rev. C 85 (2012) 034316; S. Stoica and M. Mirea, Phys. Rev. C 88 (2013) 037303.

[2] J. Hyvarinen and J. Suhonen, Phys. Rev. C 91 (2015). 024613; F. Šimkovic et al., Phys. Rev. C 87 (2013) 045501; J. Barea and F. Iachello, Phys. Rev. C 79 (2009) 044301; P. K. Rath et al., Phys. Rev. C 82 (2010) 064310; T.R. Rodriguez and G. Martinez-Pinedo, Phys. Rev. Lett. 105 (2010) 252503; J. Mendez et al., Nucl. Phys. A 818 (2009) 139.

[3] R.G.H. Robertson, Modern Phys. Lett. A 28, 1350021 (2013); S. Dell'Oro, S. Marcocci, and F. Vissani, Phys. Rev. D90, 033005 (2014).

[4] R. Arnold et al., Nucl. Inst. Meth. A 536 (2005) 79.

[5] R. Arnold et al., Eur. Phys. J. C 70 (2010) 927.

[6] J. Argyriades et al., Nucl. Inst. Meth. A 606 (2009) 449.

[7] P. Domin et al. Nucl. Phys. A753 (2005) 337-363.

[8] R. Arnold et al., Phys. Rev. Lett. 95 (2005) 182302.

[9] R. Arnold et al., Phys. Rev. D 89 (2014) 111101(R); R. Arnold et al. arXiv 1506.05825 (Accepted for publication in Phys. Rev. D).

[10] K.A. Olive et al., Particle Data Group, Chin. Phys. C 38, 090001 (2014).

[11] T. Junk, Nucl. Inst. Meth. A 434 (1999) 435. 